American J. of Engineering and Applied Sciences 2 (2): 285-291, 2009

ISSN 1941-7020

(C) 2009 Science Publications

\title{
Optical Nonlinear Proreties and Optical Limiting Effect of Metanil Yellow
}

\author{
R.K. Rekha and A. Ramalingam \\ Department of Physics, Centre for Laser Technology, Anna University, Chennai-600025, India
}

\begin{abstract}
Problem statement: The third order nonlinear optical properties of organic polymers are used in high speed telecommunication, optical limiters and optical computers. The purpose of the study was to determine the third order nonlinear characteristics and optical limiting effect of the organic dye, metanil yellow in liquid and solid medium. Approach: The samples were prepared by dissolving the dye in acetonitrile (liquid medium) at different concentration and thin film was prepared by bulk polymerization method. The nonlinear optical responses of the samples were studied by using singlebeam Z-scan technique. The optical limiting effects of the samples were studied by using a CW, $\mathrm{Nd}$ : YAG laser at $532 \mathrm{~nm}$. Results: The open aperture Z-scan of the solution and solid samples displayed reversible saturable absorption. The closed aperture Z-scan of the samples exhibited negative nonlinearity, which was larger in magnitude in solid film compared to that in solution. The third order nonlinear susceptibility was found to be 2.00 and $2.34 \times 10^{-6}$ (e.s.u) for the dye in solution and film at $0.05 \mathrm{mM}$ concentration. The saturated output power for the dye in solution and film was found to be 1.2 and $0.8 \mathrm{~mW}$ respectively at $1.0 \mathrm{mM}$ concentration. Conclusion: The dye doped in polymer can be utilized in optical limiting, low-power degenerate four wave mixing, data storage and grating applications.
\end{abstract}

Key words: Z-scan, Metanil yellow, NLO properties, optical limiting

\section{INTRODUCTION}

In the recent past, rapid technological advancements in optics have placed great demand on the development of nonlinear optical (NLO) materials with prominent applications in optical limiting and all optical switching ${ }^{[1-2]}$. Azo dye has good photo-thermal stability, dissolvability and easy preparation virtue, so it can be used as a kind of novel storage medium. The character of its molecular structure is double-bond $\mathrm{N}=\mathrm{N}$ between the two phenyls ${ }^{[3-7]}$. The third-order optical nonlinearity includes optical bleaching (i.e., saturation) or reverse saturation in the absorption aspect, whereas self-focusing or self-defocusing occurs in the refraction aspect. Of the various techniques available, Z-scan method ${ }^{[8-9]}$ is a simple and effective tool for determining nonlinear properties and is used widely in material characterization because, it provides not only the magnitudes of the real and imaginary parts of the nonlinear susceptibility, but also the sign of the real part. Optical limiting is a nonlinear optical process in which the transmittance of a material decreases with increased incident light intensity. It has been demonstrated that optical limiting can be used for pulse shaping, smoothing and pulse compression ${ }^{[10]}$. The potential applications of optical limiting devices are sensor and eye protection.
In this study, optical nonlinearity induced in dye metanil yellow by $\mathrm{CW}$ diode pumped $\mathrm{Nd}-\mathrm{YAG}$ laser with an output power of $50 \mathrm{~mW}$ at $532 \mathrm{~nm}$ was studied using Z-scan technique, based on the sample-induced changes in beam profile at the far field. The study was made for different concentrations of dye in acetonitrile and dye doped polymer film. The nonlinear refractive indices $\mathrm{n}_{2}$, nonlinear absorption coefficients $\beta$ and third order nonlinear susceptibilities $\chi^{3}$ were measured. The optical limiting behavior of the dye has been studied.

\section{MATERIALS AND METHODS}

Synthesis of dye doped polymer film: Metanil yellow, from azo dye family was chosen for the study. The molecular structure of the dye is shown in Fig. 1. ThinLayer Chromatography (TLC) test confirms the absence of any impurities in the dye. Methylmethacrylate (MMA) was chosen as a monomer for synthesizing dye doped polymer films. The modification of the polymeric material by additive solvents enhances the laser damage threshold. Spectroscopic grade acetonitrile was chosen as additive; because it combines good solubility for the Metanil yellow dye. Benzoyl peroxide was used as the initiator. The Dye Doped Polymer film (DDP) was synthesized using thermal bulk free radical polymerization method ${ }^{[11-12]}$.

Corresponding Author: R.K. Rekha, Department of Physics, Centre for Laser Technology, Anna University, Chennai-600025, India 


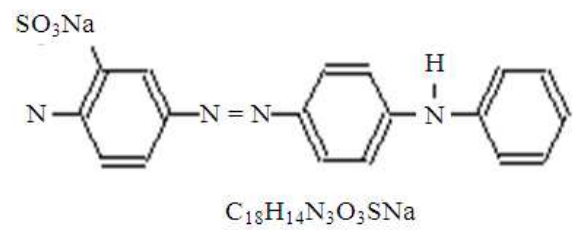

Fig. 1: Chemical structure and molecular formula of Metanil yellow

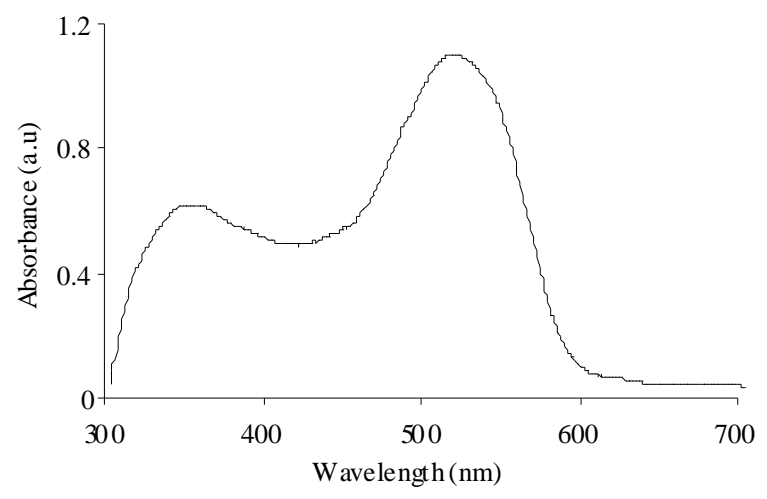

Fig. 2: UV-VIS absorption spectrum of dye Metanil yellow in acetonitrile

The optical quality of the film is checked by passing $\mathrm{He}-\mathrm{Ne}$ laser beam of power of $5 \mathrm{~mW}$. Film which shows no distortion or dispersion of the laser beam alone is taken for further studies. The DDP film of concentration $0.05 \mathrm{mM}$ and thickness of $0.9 \mathrm{~mm}$ was synthesized.

Absorption spectra: The spectral property (absorption) of the dye in liquid medium of concentration $0.01 \mathrm{mM}$ is studied by recording the absorption spectra of the dye using an UV-VIS spectrophotometer (PERKIN-EIMER LAMDA 35). The absorption spectrum of the dye is shown in Fig. 2. The spectral parameters such as absorption-peak wavelength, molar-extinctioncoefficient $\varepsilon(\lambda)$, oscillator strength (f), bandwidth $\left(\Delta v_{1 / 2}\right)$, were calculated to be $521 \mathrm{~nm}, 11.05 \times 10^{4} \mathrm{~L} \mathrm{moL}^{-1} \mathrm{~cm}^{-1}$, $2.34 \times 10^{-24} \mathrm{LmoL}^{-1} \mathrm{~cm}^{-2}$ and $4.9 \times 10^{3} \mathrm{~cm}^{-1}$ respectively.

Z-scan technique for determining the nonlinear refractive index: A diode-pumped Nd: YAG laser of wavelength $532 \mathrm{~nm}$ (Coherent Compass TM 215 M-50) is used as the excitation source for the Z-scan technique. The laser of Gaussian beam profile was focused by a convex lens, of focal length $\mathrm{f}=3.5 \mathrm{~cm}$ to produce a beam waist $\omega_{0}$ of $20.56 \mu \mathrm{m}$. The peak intensity of the incident laser beam is $\mathrm{I}_{0}=3.78 \mathrm{~kW} \mathrm{~cm}^{-2}$ and the diffraction length, $Z_{R}$ was found to be $2.5 \mathrm{~mm}$.

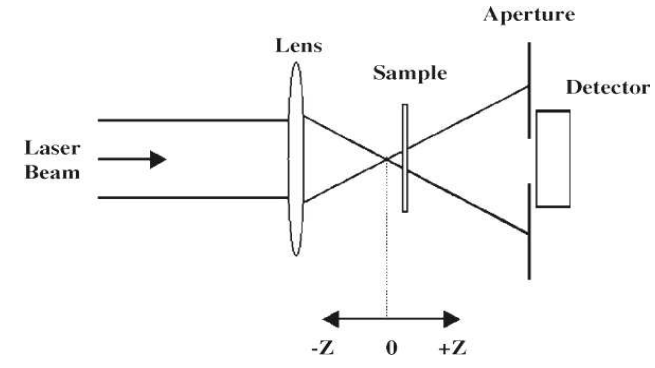

Fig. 3: Experimental setup for Z-scan

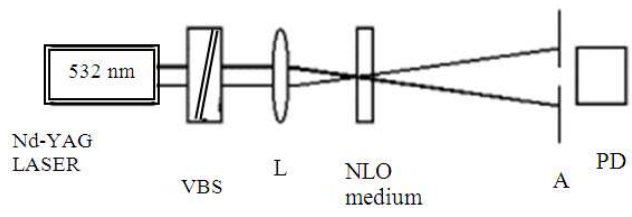

Fig. 4: Experimental setup for measuring limiting effect

The schematic diagram of the experimental set up used is shown in Fig. 3. A $1 \mathrm{~mm}$ wide optical cell containing the dye dissolved in solvent is translated across the focal region along the axial direction that is the direction of the propagation laser beam. The transmission of the beam through an aperture placed in the far field is measured using photo detector fed to the digital power meter (Field master Gs-coherent). For an open aperture Z-scan, a lens was used to collect the entire laser beam transmitted through the sample with the aperture replaced. The same was repeated for the polymer film.

Optical limiting technique: The limiting effect of the azo dye was studied by using a $50 \mathrm{~mW} \mathrm{Nd}$ : YAG CW laser at $532.8 \mathrm{~nm}$. The experimental set-up for the demonstration of optical limiting is shown in Fig. 4. A 1 -mm quartz cuvette containing dye solution is kept at the position where the transmitted intensity shows a valley in closed aperture Z-scan curve ${ }^{[8]}$. A Variable Beam Splitter (VBS) was used to vary the input power. An aperture A of variable diameter is used to control the cross-section of the beam coming out of the sample cuvette. This beam is then made to fall on the Photo Detector (PD).The input laser intensity is varied systematically and the corresponding output intensity values are measured by the photo detector.

\section{RESULTS}

The third-order nonlinear refractive index $\mathrm{n}_{2}$ and the nonlinear absorption coefficient $\beta$, of the azo dye in acetonitrile at various concentrations and the polymer 
film for the incident intensity $\mathrm{I}_{0}=3.78 \mathrm{~kW} \mathrm{~cm}{ }^{-2}$ were evaluated by the measurements of Z-scan. Figure 5a and $\mathrm{b}$ shows the open $\mathrm{Z}$-scan curve for the dye in solution at various concentration and dye doped polymer film. The defocusing effect of the dye in solution at various concentration and polymer film are shown in Fig. 6a and b.

The imaginary parts of the third-order nonlinear optical susceptibility $\left[\chi^{3}\right]$ is estimated using the value of the nonlinear absorption coefficient $\beta$ obtained from the open aperture Z-scan data.

$\beta=2 \sqrt{ } 2 . \Delta \mathrm{T} / \mathrm{I}_{0} \mathrm{~L}_{\text {eff }}$

where, $\Delta \mathrm{T}$ is the normalized transmittance of the sample when at position $\mathrm{Z}$.
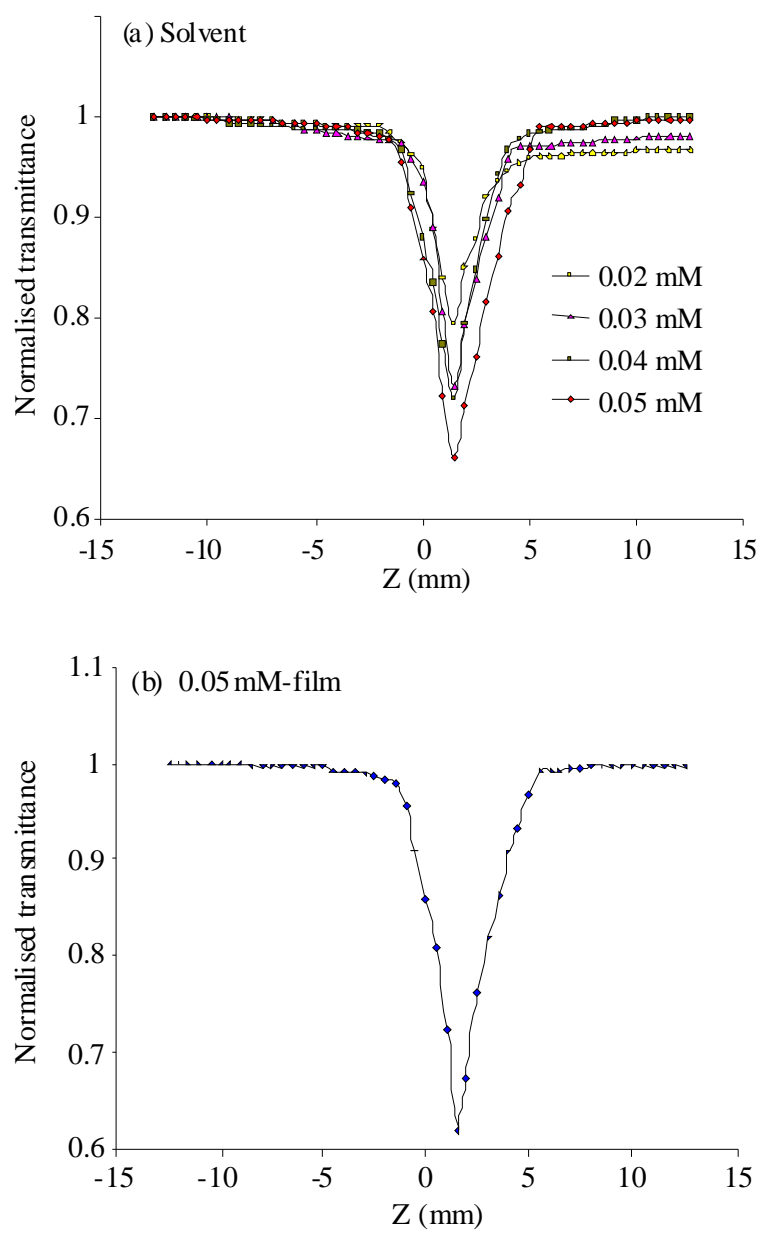

Fig. 5: Open z scan curve (a): Dye in solvent at various Concentration; (b): Dye doped polymer film
The on-axis phase shift $\Delta \phi_{0}$ at the focus is related to the third order nonlinear refractive index $\left(\mathrm{n}_{2}\right)$ by:

$$
\Delta \phi_{\mathrm{o}}=\mathrm{kn}_{2} \mathrm{~L}_{\mathrm{eff}} \mathrm{I}_{\mathrm{o}}
$$

Where:

$\mathrm{I}_{0}=$ The intensity of the laser beam at focus $\mathrm{z}=0$

$\mathrm{k}=$ The wave number $(\mathrm{k}=2 \pi / \lambda)$

$\lambda=$ The wavelength of the light used

$\mathrm{L}_{\text {eff }}=\left(1-\mathrm{e}^{-\alpha \mathrm{L}}\right) / \alpha$

$=$ The effective thickness of the sample

$\mathrm{L}=$ The thickness of the sample

$\alpha=$ The linear absorption coefficient

Experimentally determined nonlinear refractive index $\mathrm{n}_{2}$ and nonlinear absorption coefficient $\beta$ can be used in finding the real and imaginary parts of the thirdorder nonlinear optical susceptibility $\left[\chi^{3}\right]^{[13]}$ according to the following relations:

$$
\operatorname{Re} \chi^{3}(\mathrm{esu})=10^{-4} \frac{\varepsilon_{\mathrm{o}} \mathrm{c}^{2} \mathrm{n}_{\mathrm{o}}{ }^{2}}{\pi} \mathrm{n}_{2}\left(\frac{\mathrm{cm}^{2}}{\mathrm{~W}}\right)
$$
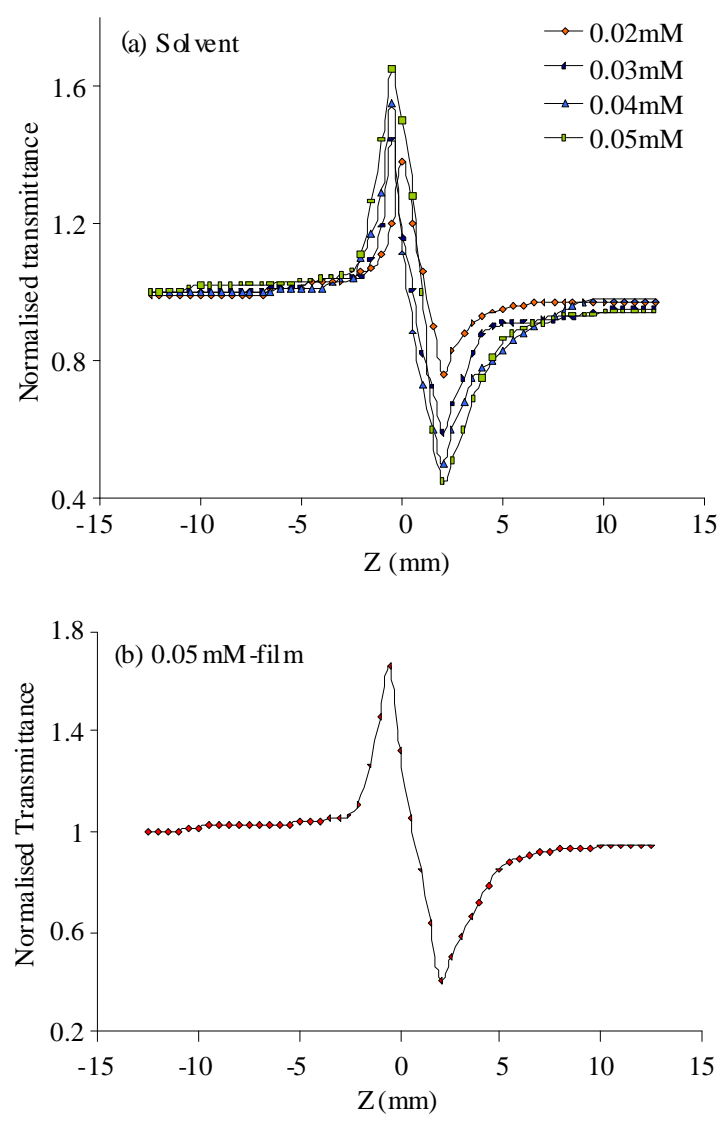

Fig. 6: Closed z scan curve (a): Dye in solvent at various concentration; (b): Dye doped polymer film 
Am. J. Engg. \& Applied Sci., 2 (2): 285-291, 2009

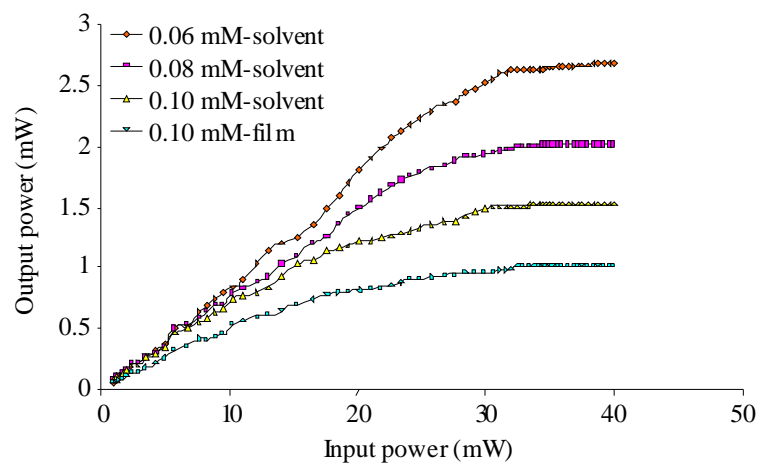

Fig. 7: Optical limiting effects of Metanil yellow dye in solvent and dye doped polymer film

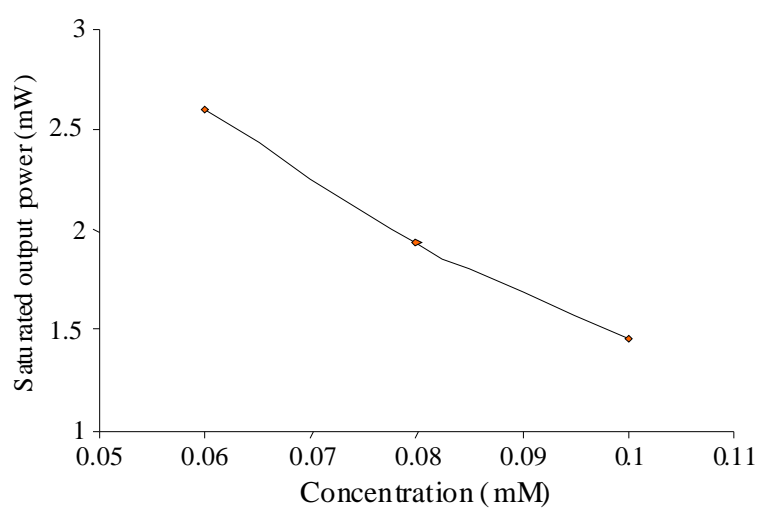

Fig. 8: Concentration dependence of saturated output value of Metanil yellow dye in acetonitrile

$\mathrm{I}_{\mathrm{m}} \chi^{3}(\mathrm{esu})=10^{-2} \frac{\varepsilon_{\mathrm{o}} \mathrm{c}^{2} \mathrm{n}_{\mathrm{o}}{ }^{2} \lambda}{4 \pi^{2}} \beta\left(\frac{\mathrm{cm}}{\mathrm{W}}\right)$

Where:

$\varepsilon_{0}=$ The vacuum permittivity

$\mathrm{c}=$ The light velocity in vacuum

The absolute value of the third-order nonlinear optical susceptibility is given by the relation:

$\left|\chi^{3}\right|=\left[\left(\mathrm{R}_{\mathrm{e}}\left(\chi^{3}\right)\right)^{2}+\left(\mathrm{I}_{\mathrm{m}}\left(\chi^{3}\right)\right)^{2}\right]^{1 / 2}$

The experiment was repeated for the pure solvent (acetonitrile) to account for its contribution, but no significant measurable signals were produced in either the open or the closed Z-scan traces. The nonlinear parameters calculated are as tabulated in Table 1.

The optical limiting behavior of metanil yellow for different concentration in solution and dye doped polymer film are shown in Fig. 7. The Saturated output value at which the limiting occurs for the dye in solvent is shown in Fig. 8 for different concentration.
Table.1. Nonlinear parameters-Metanil yellow dye in acetonitrile

\begin{tabular}{llllll}
\hline Concentration & $\Delta \mathrm{T}_{\mathrm{p}-\mathrm{v}} \mathrm{n}_{2} \times 10^{-8}$ & $\begin{array}{c}\chi \times 10^{-3} \\
\mathrm{~cm} \mathrm{~W}^{2} \mathrm{~W}^{-1}\end{array}$ & $\begin{array}{l}\mathrm{L} \times 10^{-4} \\
\text { (e.s.u) }\end{array}$ \\
\hline Liquid medium & & & & & \\
$0.02 \mathrm{mM}$ & 0.62 & -2.33 & 0.93 & -1.58 & 1.03 \\
$0.03 \mathrm{mM}$ & 0.86 & -3.23 & 1.21 & -2.19 & 1.44 \\
$0.04 \mathrm{mM}$ & 1.06 & -4.00 & 1.35 & -2.71 & 1.77 \\
$0.05 \mathrm{mM}$ & 1.20 & -4.51 & 1.53 & -3.06 & 2.00 \\
Polymer film (0.05 mM) & 1.26 & -5.27 & 1.88 & -3.57 & 2.34 \\
\hline
\end{tabular}

\section{DISCUSSION}

Z-scan studies: The metanil yellow azo dye studied, displayed two absorption bands in the range 300-650 $\mathrm{nm}$, being transparent in the near infrared region as observed from the Fig. 2. Localized absorption of a tightly focused beam propagating through an absorbingdye medium produces a spatial distribution of temperature in the dye solution and consequently, a spatial variation of the refractive index that acts as a thermal lens resulting in the phase distortion of the propagating beam. Z-scan with a fully open aperture $(S=1)$ is insensitive to nonlinear refraction (thin sample approximation). $\mathrm{S}$ the aperture linear transmittance is given by:

$S=1-\exp \left(-2 r^{2} a / \omega_{a}^{2}\right)$

With:

$\mathrm{r}_{\mathrm{a}}=$ Denoting the aperture radius

$\omega_{\mathrm{a}}=$ Denoting the radius of the laser spot before the aperture

Such Z-scan traces with no aperture are expected to be symmetric with respect to the focus $(Z=0)$ where they have a minimum transmittance (Reversible saturation absorption). The peak followed by a valleynormalized transmittance curve obtained from the closed aperture Z-scan data, indicates that the sign of the refraction nonlinearity is negative (i.e.) selfdefocusing. The self-defocusing effect is due to the local variation of the refractive index with temperature. The measurable quantity $\Delta \mathrm{T}_{\mathrm{p}-\mathrm{v}}$ can be defined as the difference between the normalized peak and valley transmittances, $T_{p}-T_{v}$. The variation of this quantity ${ }^{[14]}$ as a function of $\left|\Delta \phi_{\mathrm{o}}\right|$ is given by:

$\Delta \mathrm{T}_{\mathrm{p}-\mathrm{v}}=0.406(1-\mathrm{S})^{0.25}\left|\Delta \phi_{\mathrm{o}}\right|$

If we collect all the energy transmitted by the sample (open-aperture Z-scan), the measurement is sensitive to nonlinear absorption only. If an aperture is placed in front of the detector (closed-aperture Z-scan), the measurement is sensitive to both nonlinear 
Am. J. Engg. \& Applied Sci., 2 (2): 285-291, 2009
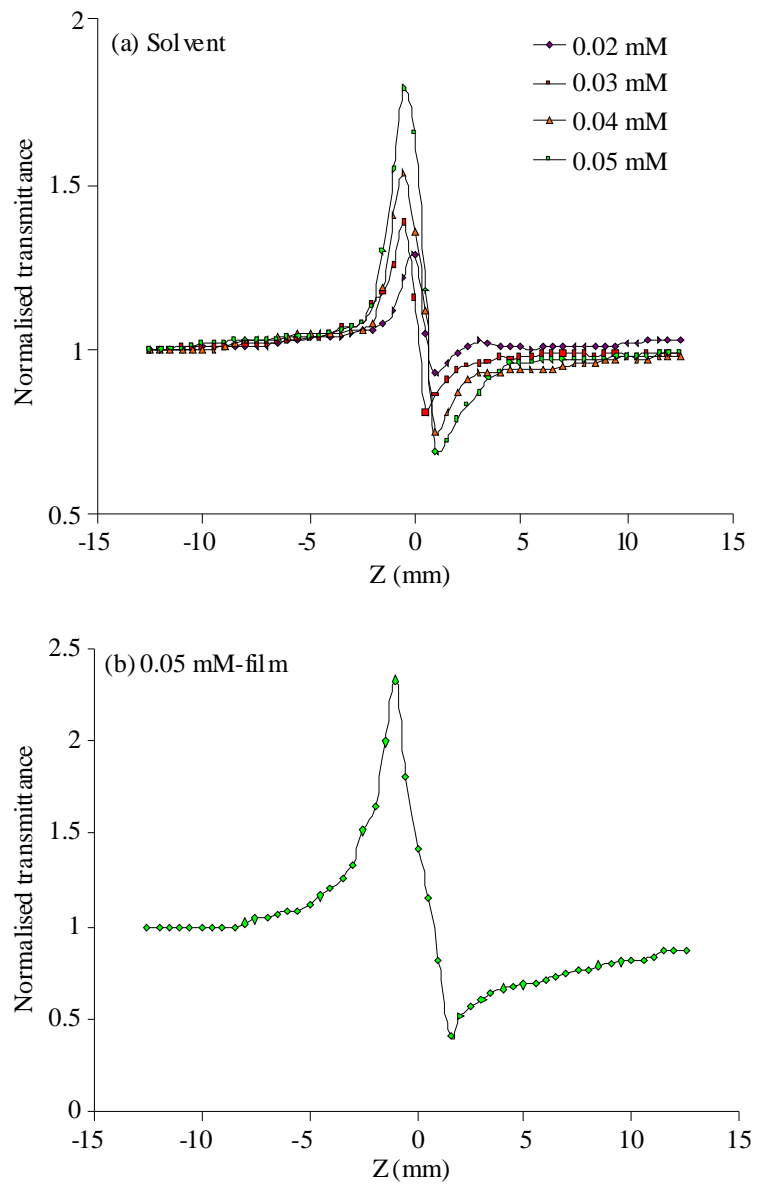

Fig 9: Pure nonlinear refraction curve (a): Dye in solvent at various concentration; (b): Dye doped polymer film

absorption and nonlinear refraction. To get the pure nonlinear refraction contribution, we divide the closedaperture Z-scan transmittance by the one with open aperture $^{[9]}$ and the experimental curves are shown in Fig. 9a and $\mathrm{b}$ for dye in solvent and film.

The value of $\Delta \mathrm{T}_{\mathrm{p}-\mathrm{v}}$ has increased for the dye doped polymer film when compared to the dye in acetonitrile. This may be due to the heat dissipation being faster in liquids as compared to that in a solid medium. From Fig 10a-c there is an increasing trend for the values of $\mathrm{n}_{2}, \beta$ and $\chi^{3}$ as the concentration increases. This may be attributed to the fact that, as the number of dye molecules increases when concentration increases, more particles are thermally agitated resulting in an enhanced effect. The optical nonlinearity of the dye may be due to laser heating induced nonlinear effect. A laser beam, while passing through an absorbing media, induces temperature and density gradients that change

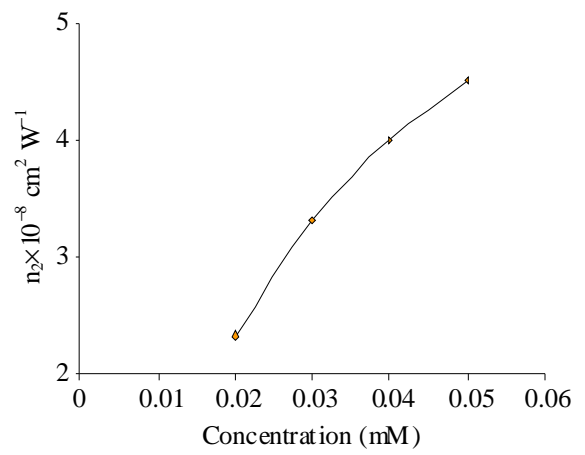

(a)

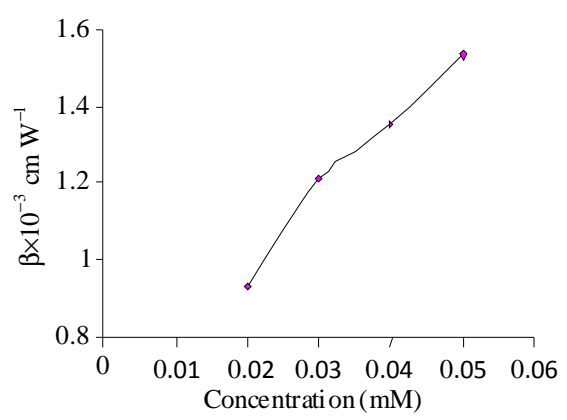

(b)

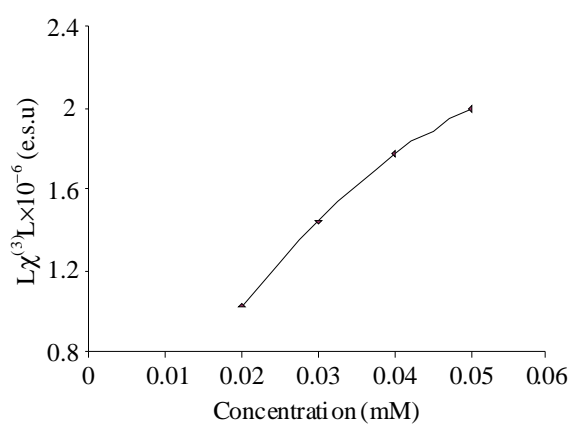

(c)

Fig. 10: Concentration dependence of (a): $\mathrm{n}_{2} ;(\mathrm{b})$ : $\beta$; (c): $\chi^{3}$ of Metanil yellow dye in acetonitrile

the refractive index profile. This intensity-induced localized change in the refractive index results in a lensing effect on the optical beam

Optical limiting: From Fig. 7 the transmitted output intensity is found to vary linearly with the incident input intensity at very low input intensities, but starts to deviate at high incident intensities. With further increment of the input power, the transmitted intensity reaches a plateau and is saturated at a point defined as the limiting amplitude. i.e., the maximum output intensity, showing obvious limiting property. Since the samples are pumped with $\mathrm{CW}$ laser beam the arising nonlinearities are predominantly thermal in nature. 
The UV-visible absorption spectrum of the samples before and after the laser irradiation shows that the pattern and intensity of the spectrum does not show any change, indicating that the samples possesses good photostability. The dyes investigated here are very weakly fluorescent and is non fluorescent at the wavelength studied, optimizing the conversion of the absorption energy into heat ${ }^{[15]}$. The limiting effect depends on the concentration and increases with increases in concentration as shown in Fig. 8. The experiment was repeated for the pure solvent (acetonitrile) to account for its contribution, but no limiting was observed. The results were comparable to some of the recent reports of low power optical limiting ${ }^{[16]}$. So; the sample possesses limiting effect for the light of $532 \mathrm{~nm}$.

\section{CONCLUSION}

The nonlinear absorption and refraction in the organic dye, both in solution and solid film were studied using a single-beam Z-scan technique under $\mathrm{CW}$ laser with excitation at $532 \mathrm{~nm}$. It is worth noting that the value of $\chi^{3}$ for the dye studied is larger than those of some representative third-order nonlinear optical materials such as chalcone and its derivatives and organic dyes like Croconium ${ }^{[17-18]}$. It is found that the observed nonlinear absorption is caused by a reversible saturation absorption process. These results are quite encouraging for possible applications in nonlinear optical devices. Limiters based on nonlinear absorption phenomena have been designed for use with high power pulsed laser sources, but limiters demonstrated based on photo chromic-thermo optic nonlinearity such as the one studied here can be used as efficient limiters in the $\mathrm{CW}$ regime.

\section{ACKNOWLEDGEMENT}

The researchers wish to thank the DAE-BRNS for their financial support.

\section{REFERENCE}

1. Venkatram, N., D. NarayanaRao, L. Giribabu and S. VenugopalRao, 2008. Femtosecond nonlinear optical properties of alkoxy phthalocyanines at $800 \mathrm{~nm}$ studied using Z-scan technique. Chem. Phys. Lett., 464: 211-215. DOI: 10.1016/j.cplett.2008.09.029

2. Huang, T., Z. Hao, H. Gong, Z. Liu, S. Xiao and S. Li et al., 2008. Third order nonlinear optical properties of a new copper coordination compound: A promising candidate for all optical switching. Chem. Phys. Lett., 451: 213-217. DOI: 10.1016/j.cplett.2007.12.001
3. Qi, S., C. Zhang, X. Yang, K. Chen, L. Zhang and G. Yang et al., 2006. Absorption characteristic and limiting effect of Congo red doped PVA film. Opt. Mater., $\quad 11$ : 1348-1351. $\quad$ DOI: 10.1016/j.optmat.2006.06.015

4. Qi, S., C. Zhang, X. Yang, K. Chen, L. Zhang and Y. Liu et al., 2007. Effective indexes of refraction and limiting properties of ethyl red. Optik, 118: 425-429. DOI: 10.1016/j.ijleo.2006.03.029

5. Yang, X., C. Zhang, S. Qi, K. Chen, J. Tian and G. Zhang, 2005. All- optical Boolean logic gate using azo-dye doped polymer film. Optik, 116: 251-254. DOI: 10.1016/j.jileo.2005.01.017

6. Gayathri, C. and A. Ramalingam, 2008. Investigation of optical nonlinearities of an azo dyes using a $532 \mathrm{~nm}$ diode-pumped Nd: YAG laser. Spectrochima Acta Part A., 69: 96-99. DOI: 10.1016/j.saa.2007.031.025

7. Qi, S., X. Yang, R. Lu, K. Chen, C. Zhang, J. Tian, J. Xu and Q. Wu, 2004. Nonlinear optical properties of mercury dithizonate in a polymer film. J. Modern Opt., 51: 1671-1677. DOI: 10.1080/09500340410001664700

8. Sheik-Bahae, M., A.A. Said and E.W. Van Stryland, 1989. High-sensitivity, single-beam $\quad \mathrm{n}_{2}$ measurements. Opt. Lett., 14: 955-957. DOI: 10.1364/OL.14.000955

9. Sheik-Bahae, M., A.A. Said, T.H. Wei, D.J. Hagan and E.W. Van Stryland, 1990. Sensitive measurement of optical nonlinearities using a single beam IEEE. J. Quant. Elect., 26: 760-769. DOI: $10.1109 / 3.53394$

10. Qu, S., C. Zhao, X. Jiang, G. Fang, Y. Gao and H. Zeng et al., 2003. Optical nonlinearities of space selectively precipitated $\mathrm{Au}$ nanoparticles inside glasses. Chem. Phys. Lett., 368: 352-358. DOI: 10.1016/S0009-2614(02)01885-7

11. Costela, A., I. GarciaMarino, J.M. Figuera, F. Amat-Guerri, J. Barroso and R. Sastre, 1996. Solid-state dye laser based on Coumarin 540Adoped polymeric matrices. Opt. Commun., 130: 44-50. DOI: 10.1016/0030-4018(96)00220-9

12. SindhuSukumaran, V. and A. Ramalingam, 2006. Spectral characteristics and nonlinear studies of crystal violet dye. Spectrochim. Acta Part A., 63: 673-676. DOI: 10.1016/j.saa.2005.06.017

13. Cassano, T., R. Tommasi, M. Ferrara, F. Babudri, G.M. Farinola and F. Naso, 2001. Substituentdependence of the optical nonlinearities in poly (2,5-dialkoxy-p-phenylenevinylene) polymers investigated by the Z-scan technique. Chem. Phys., 272: $\quad 111-118 . \quad$ DOI: $10.1016 / \mathrm{S} 0301-$ 0104(01)00453-0 
14. Mathews, S.J., S. Chaitanya Kumar, L. Giribabu and S. Venugopal Rao, 2007. Nonlinear optical and optical limiting properties of phthalocyanines in solution and thin films of PMMA at $633 \mathrm{~nm}$ studied using a cw laser. Mater. Lett., 61: 4426-4431. DOI: 10.1016/j.matlet.2007.02.034

15. Rashidian, M., D. Dorranian, S. Ahmadi Darani, S. Saghafi and M. Ghoranneviss, 2008. Nonlinear responses and optical limiting behavior of Basic Violet 16 dye under $\mathrm{CW}$ laser illumination. OPTIK. DOI: 10.1016/j.ijleo.2008.05.001

16. Sendhil, K., C. Vijayan and M.P. Kothiyal, 2006. Low-threshold optical power limiting of $\mathrm{cw}$ laser illumination based on nonlinear refraction in zinc tetraphenyl porphyrin. Opt. Laser Technol., 38: 512-515. DOI: $10.1016 /$ j.optlastec.2004.12.005
17. Ravindra, H.J., A. JohnKiran, K. Chandrasekaran, H.D. Shashikala and S.M. Dharmaprakash, 2007. Third order nonlinear optical properties and optical limiting in donor/acceptor substituted 4'-methoxy chalcone derivatives. Applied Phys. B (Laser Opt.), 88: 105-110. DOI: 10.1007/s00340-007-2677-8

18. Li, Z., Z.H. Jin, K. Kasatani, H. Okamoto and S. Takenaka, 2005. Large third-order optical nonlinearities of a croconium dye in tetrahydrofuran solution and in poly (methyl methacrylate) coating films measured using resonant femtosecond degenerate four-wave mixing technique. Jap. J. Applied Phys., 44: 4956-4961. DOI: 10.1143/JJAP.44.4956 